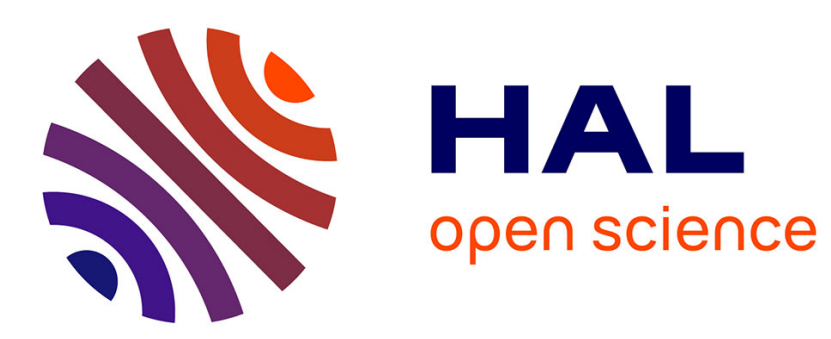

\title{
Travail et famille dans un village du Hebei
}

\author{
Isabelle Thireau, Kong Mak
}

\section{To cite this version:}

Isabelle Thireau, Kong Mak. Travail et famille dans un village du Hebei. Études Chinoises, 1989, 8 (1), pp.7-40. halshs-00179405

\section{HAL Id: halshs-00179405 https://shs.hal.science/halshs-00179405}

Submitted on 15 Oct 2007

HAL is a multi-disciplinary open access archive for the deposit and dissemination of scientific research documents, whether they are published or not. The documents may come from teaching and research institutions in France or abroad, or from public or private research centers.
L'archive ouverte pluridisciplinaire HAL, est destinée au dépôt et à la diffusion de documents scientifiques de niveau recherche, publiés ou non, émanant des établissements d'enseignement et de recherche français ou étrangers, des laboratoires publics ou privés. 


\section{Travail et famille dans un village du Hebei

\author{
Isabelle Thireau \\ Mak Kong ${ }^{1}$
}

La création d'industries rurales, aussi bien privées que placées sous la direction des collectivités locales, a été encouragée en Chine depuis le début des années quatre-vingt. Cette nouvelle stratégie visait à la fois à soutenir le développement économique des campagnes chinoises et à résoudre le problème de l'excédent de main-d'œuvre agricole à travers une diversification des activités de production ${ }^{2}$.

Si les effets du processus d'industrialisation ainsi amorcé se révèlent très inégaux suivant les régions, il n'en demeure pas moins que des zones autrefois exclusivement rurales ont vu soudain se multiplier commerces et petites ou moyennes industries. Des villages dont l'économie reposait jusque-là sur des activités exclusivement agricoles ont créé leurs propres entreprises ou envoyé une partie de leur population active s'embaucher dans les usines environnantes.

1. Is abelle Thireau est chargée de recherches au CNRS ; Mak Kong prépare une thèse sur la Révolution culturelle à l'École des Hautes Études en Sciences Sociales.

2. La main d'œuvre excédentaire était estimée en 1987 à cent millions de personnes. Cf. «Zhongguo nongcun chanye jiegou yanjiu baogao » (Rapport de recherche sur la structure des activités de production dans les villages chinois), Zhongguo nongcun jingji, 1987, 7, pp. 1-7.

Études chinoises, vol. VIII, $\mathrm{n}^{\circ} 1$, printemps 1989 
Le groupe familial est profondément affecté par cette évolution des structures économiques. Progressivement une séparation s'instaure entre la famille et les activités professionnelles de chacun. La décollectivisation et l'essor industriel appellent de nouvelles négociations pour redéfinir les échanges familiaux et exploiter au mieux les ressources économiques et sociales du groupe. Le travail commence à sortir de la famille et de nombreux foyers paysans accèdent ainsi à la phase de transition qui sépare, selon Alain Girard, le monde pré-industriel de la société industrielle ${ }^{3}$.

Comment s'opère plus particulièrement ce changement en Chine ? Quelle articulation peut-on observer entre les nouvelles activités non agricoles de production et les conduites sociales liées au groupe familial ? Nous avons, pour répondre à ces questions, effectué en octobre 1986 un court séjour dans un village situé à 60 kilomètres de Pékin, village dont nous savions seulement qu'il était le siège, depuis quelques années, d'un important phénomène d'industrialisation, et qui semblait donc a priori convenir à une étude de l'évolution des structures familiales paysannes au cours de la première phase de développement industriel.

Étant donné le temps très court dont nous disposions pour l'enquête sur le terrain, nous avions établi un questionnaire préalable que nous remplissions au cours d'un entretien avec un ou plusieurs membres de chacune des familles étudiées. Les questions posées se rapportaient essentiellement au fonctionnement économique des maisonnées, l'accent étant mis sur les nouvelles interactions familiales qui s'expriment dans les domaines de l'emploi, de la rémunération, de la distribution des biens ou du partage du pouvoir décisionnel en matière financière. En effet ces thèmes se prêtent assez volontiers à l'usage du questionnaire, alors que cette méthode aurait été d'application beaucoup plus difficile pour étudier, par exemple, l'évolution des valeurs culturelles ou des normes morales au sein du groupe domestique.

3. Cf. Alain Girard, «D'un monde à l'autre : du travail commun dans la famille à l'activité professionnelle hors du foyer ", La revue Tocqueville, vol. 5 , 1983, pp. 419-428. 
Vingt-deux familles choisies au hasard ${ }^{4}$ ont accepté de répondre à notre questionnaire et de nous accueillir chez elles pour un entretien ${ }^{5}$. Les données ainsi recueillies, complétées par des discussions avec les cadres locaux, constituent les principales sources de l'analyse présentée ici.

\section{Baozi Pu : du village à l'usine}

Baozi Pu, le village étudié, appartient au district de Zuo, l'un des vingtdeux qui composent la région de Baoding (Hebei) ${ }^{6}$. Zuo connaît une certaine renommée dès le $\mathrm{III}^{c}$ siècle de notre ère. Plusieurs personnages célèbres des Trois Royaumes (220-265) y ont en effet vu le jour, et les hauts faits de ces héros constituent aujourd'hui encore un thème privilégié des conversations villageoises. Bien que les temples dédiés à Zhang Fei et à Liu Bei aient été détruits au moment de la Révolution culturelle, les paysans aiment à évoquer les fêtes qui s'y déroulaient, et seul le maintien d'un contrôle politique assez étroit semble empêcher que ces édifices soient restaurés.

Le village de Baozi Pu existait déjà sous la dynastie des Qing puisqu'on y trouvait un relais de poste. Malgré la proximité de la capitale, la région est demeurée essentiellement agraire jusqu'à l'arrivée au pouvoir du Parti communiste chinois. Le blé et le maïs étaient les deux cultures dominantes pendant la première moitié du siècle, et deux artisanats traditionnels paraissent avoir été très développés : le tissage manuel

4. Les deux enquêteurs, auteurs du présent article, ont choisi chacun une ruelle du village, passant outre les maisons où il n'y avait que des enfants pour les recevoir.

5. Ces vingt-deux familles, qui regroupent environ un septième de la population du village, semblent assez représentatives de la communauté étudiée puisque leur revenu par personne était de 1230 yuans en 1985, contre 1240 yuans pour l'ensemble du village.

6. Notons au passage que Hu Yaobang, recevant à Pékin en janvier 1985 les responsables de ces vingt-deux districts, les félicita pour la hausse du revenu par habitant dans les zones rurales obtenue entre 1983 (243 yuans) et 1984 (344 yuans). Cf. Renmin ribao, 7 janvier 1985. 
de tapis et la vannerie. L'un et l'autre disparaissent avec l'avènement des communes populaires en 1958. Baozi Pu devient alors une des brigades de production de la commune de Chengguan, le village étant lui-même divisé en quatre équipes de production. Aujourd'hui, comme pendant la période collectiviste, les principales cultures sont le blé, le maïs et le millet. Haricots et arachides occupent également une petite superficie.

Les activités subsidiaires étant interdites, le village ne comptait ni commerces ni entreprises avant le début des récentes réformes. Seuls quelques paysans, protégés par les cadres locaux, étaient parvenus à se faire embaucher dans les services administratifs ou dans les commerces regroupés au chef-lieu du district.

Entre 1978 et 1985, le niveau de vie des habitants de Baozi Pu a connu une amélioration d'une rapidité sans précédent. Le revenu annuel par habitant est en effet passé de 184 yuans en 1978 à 1240 yuans en 1985. L'achat massif de biens d'équipement et de biens de consommation, depuis 1980 et surtout au cours des années 1985-1986, confirme cet enrichissement. Ainsi, avant 1979 une seule famille parmi les vingt-deux que nous avons interrogées avait fait l'acquisition d'un poste de télévision, alors qu'elles en possédaient toutes en octobre 1986. A cette date on comptait également 14 magnétophones, 11 machines à laver, 7 réfrigérateurs, 5 motos et 2 machines à coudre. La totalité des motos et des machines à coudre, ainsi que 10 des 11 machines à laver, avaient été achetées après 1984.

Vingt-deux nouvelles maisons ont été construites depuis les réformes par les foyers ayant répondu à notre questionnaire, soit une par famille en moyenne. Ces maisons représentent 80 pièces au total, et pour près de la moitié (37) les travaux datent de 1985. Certains foyers ont même fait installer l'eau courante. Le mode de vie a également changé. Par exemple, grâce à l'augmentation des ressources, les habitants du village peuvent se rendre à Pékin beaucoup plus souvent qu'autrefois. Aller passer quelques jours à la capitale est en effet cité aujourd'hui comme la principale activité de loisir des jeunes célibataires. "La région et le village ", remarquait un paysan pendant notre séjour, « sont méconnaissables par rapport à la situation que nous connaissions vers la fin des années 
soixante-dix. Jamais nous n'avions rêvé d'une telle prospérité. Bien des citadins envient aujourd'hui notre revenu... »'.

Pourtant, seules quelques communautés dans la région peuvent se prévaloir d'une augmentation aussi rapide de leur niveau de vie. Le revenu annuel moyen par habitant des zones rurales de la province n'était en effet que de 401 yuans en 1986, et en 1984, 23 villages seulement parmi les quelque 5000 que compte la région de Baoding jouissaient d'un revenu annuel supérieur à 1000 yuans par habitant ${ }^{8}$.

La croissance économique exceptionnelle de Baozi Pu n'est pas liée au développement des activités agricoles, qui apparaissent au contraire en déclin. L'essor d'un nombre important d'entreprises et de commerces privés dans le district de Zuo explique en partie cette réussite. Mais une augmentation aussi rapide du niveau de vie n'aurait pu avoir lieu sans la création, en 1980, d'une usine au sein même du village étudié.

\section{Le déclin des activités agraires et l'essor industriel}

1980 est aussi l'année où les responsables de Baozi Pu ont procédé au premier mouvement de redistribution des moyens de production. Si chaque villageois avait reçu en moyenne 2 mou de terre lors de la réforme agraire', du fait de l'augmentation de la population au cours des trois dernières décennies 1,2 mou seulement ont pu être attribués à chaque travailleur en 1980 .

Une seconde répartition des terres est intervenue en 1985, des contrats d'exploitation étant alors conclus avec les différentes maisonnées. Le développement de nouvelles activités de production jouant un rôle prééminent dans l'économie locale a cependant incité les cadres villageois à proposer de réduire encore la superficie des terres destinées aux

7. Entretien, Baozi Pu, 8 octobre 1986.

8. Dans plusieurs centaines de villages de la région de Baoding le revenu annuel moyen ne dépassait pas 100 yuans par personne en 1984. Cf. Hebei ribao, 20 février 1985 (Survey of World Broadcasts, 8 avril 1985).

9. Un mou équivaut à un quinzième d'hectare. 
groupes domestiques et de créer un jardin potager collectif. Ce projet, qui permettait aux habitants de Baozi Pu d'orienter une partie de la maind'œuvre familiale vers des tâches plus lucratives, a été accepté. Chacun a reçu ainsi le droit d'exploiter 0,8 mou de terre, et ce pour une période de quinze ans ${ }^{10}$.

Quelles que soient l'étendue des terres attribuées et la hausse des rendements résultant d'une intensification des soins apportés aux champs, à elles seules les activités agricoles ne pouvaient procurer qu'un revenu relativement faible aux habitants de Baozi Pu. En 1985, pour ne donner que deux exemples, une famille ayant obtenu l'usufruit de $3 \mathrm{mou}$ de terre a gagné 650 yuans grâce à la vente d'un cochon et à celle de sa production de céréales à l'État ; et les activités agricoles d'une famille de trois personnes à laquelle avait été attribuée une parcelle de 2,4 mou ont rapporté un peu moins de 400 yuans, soit 133 yuans par personne. La part des récoltes consommée sur place n'est guère importante et ne représente qu'un faible pourcentage du revenu paysan. Les 22 familles qui composent notre échantillon ont ainsi l'usufruit d'une superficie globale de 76,1 mou de terre cultivable, soit une moyenne de 3,5 mou par foyer. Seuls 3,3 mou dans ce total sont utilisés pour la culture des légumes, la plupart des familles préférant acheter les produits du jardin potager collectif. Une fois vendu à l'État le montant des récoltes fixé au préalable, et une fois les impôts versés, le reste de la production permet de nourrir les 104 personnes concernées. En revanche les paysans sont obligés d'acheter du riz ou du blé pour nourrir la main-d'œuvre extérieure à la maisonnée qui travaille et loge sur place dans le cadre des industries domestiques que nous décrirons plus loin.

En d'autres termes, les 704 habitants du village, disposant d'une attribution totale de $550 \mathrm{mou}$, n'auraient pu connaître de véritable prospérité sans une profonde transformation des activités de production. De fait, le processus d'industrialisation qui a débuté de façon soudaine à

10. Lors du premier mouvement de distribution, les champs avaient été attribués en fonction du nombre de membres actifs au sein de chaque famille. En 1985, les terres étant réparties pour une période beaucoup plus longue, la taille des parcelles a été calculée d'après le nombre total de personnes par groupe domestique. 
Baozi Pu a eu pour effet de reléguer les travaux des champs au dernier rang des sources locales de revenu. Ainsi, en 1985, les ressources tirées de la vente des produits agricoles ne représentaient que $6,6 \%$ du revenu monétaire moyen des 22 maisonnées auprès desquelles l'enquête a été menée. En revanche $62 \%$ du revenu familial provenait de la vente des tapis fabriqués artisanalement dans des ateliers domestiques et vendus à l'usine collective récemment créée à Baozi $\mathrm{Pu}$.

C'est en effet en 1980, lors du premier mouvement de redistribution des terres, que quelques cadres de la brigade, tous membres du Parti, prennent une initiative qui va avoir de profondes répercussions sur le développement économique du village. Au lieu de répartir entre les familles paysannes les équipements acquis par la brigade sous le système collectiviste (comme dans la plupart des autres villages), ils proposent de remettre à l'honneur un des artisanats traditionnels de la région : le tissage manuel de tapis. Leur projet est de créer un atelier collectif de fabrication de tapis, auquel seront confiés les cinq petits camions et le tracteur appartenant à la brigade. L'idée est adoptée par les villageois et quelques bâtiments, appartenant également à la collectivité, accueillent les premiers métiers à tisser, achetés grâce à des investissements personnels. Les cadres de la brigade concluent un contrat avec les services commerciaux du district de Zuo. Ces derniers leur fourniront la laine et les motifs, et l'atelier leur vendra les produits finis destinés à l'exportation.

S'il ne nous a pas été possible d'obtenir de plus amples renseignements sur les conditions ayant permis aux cadres locaux de concrétiser aussi aisément leur projet, il est clair que la famille du paysan qui dirige à la fois l'usine et le village bénéficie de liens privilégiés avec des cadres influents du district. Son père dirige en effet, lui aussi, une usine de fabrication de tapis, celle-là placée directement sous le contrôle du gouvernement du district, située à quelques kilomètres de $\mathrm{Baozi} \mathrm{Pu}$; cette usine travaille avec quelque vingt mille foyers répartis dans quatre provinces différentes. Une petite dynastie est ainsi en train de se créer, qui préside aux destinées de plusieurs milliers de paysans ${ }^{11}$.

11. Nous avons visité cette usine et nous sommes entretenus avec son responsable le 10 octobre 1986. 
L'entreprise de Baozi $\mathrm{Pu}$, où ne travaillaient au départ que quelques parents et amis des responsables locaux à l'origine de l'initiative, a prospéré très vite. Dès 1982 elle était en mesure de former des techniciens qui réalisaient désormais les motifs sur place.

En 1985, l'organisation administrative en communes populaires, brigades et équipes de production disparaît, l'ancien système des villages naturels étant restauré. L'usine de Baozi Pu est alors placée sous la direction du département du commerce extérieur de la province du Hebei, lequel fixe un quota de production annuel. Elle s'est agrandie rapidement au cours de ces dernières années puisqu'elle employait 300 personnes en octobre 1986, 120 étant affectées au travail de tissage. Mais l'originalité de l'entreprise réside surtout dans le fait que près de 2700 personnes travaillent pour elle en sous-traitance dans le cadre d'ateliers domestiques établis à Baozi Pu et dans les villages environnants. 140 des 154 foyers du village étudié possèdent des métiers à tisser ; ils reçoivent de l'usine de Baozi Pu laine et motifs, tissent les tapis, puis les vendent à l'usine qui se charge des travaux de finition, lavage, séchage, etc.

Ce modèle de développement économique était encouragé par les autorités au moment de notre enquête et il n'a pas été officiellement remis en cause depuis ${ }^{12}$. Dix entreprises de ce type existaient en juillet 1985

12. Cf. Xing Congzhi, « Lingdao qunzhong zhifu yu jianchi shehuizhuyi fangxiang » (Diriger les masses vers la prospérité et persister dans la voie socialiste), Hongqi, 1985, 8, pp. 20-22. Cela dit, les attaques récentes contre la libéralisation bourgeoise pourraient bien avoir des conséquences néfastes sur le développement économique de Baozi Pu. En effet, depuis le mois de juin 1989, et en dépit des affirmations réitérées des dirigeants chinois selon lesquelles la politique d'ouverture et de réforme se poursuit, la campagne contre la « libéralisation bourgeoise » $s$ ' accompagne de critiques de plus en plus violentes à l'encontre de la propriété privée. Le 30 août 1989, par exemple, un communiqué (en anglais) de l'agence Xinhua reprenait le contenu d'un article paru quelques jours plus tôt dans le Jingji ribao qui disait notamment : « Le socialisme doit rejeter les pratiques capitalistes de la propriété privée, de l'exploitation des ouvriers et de la distribution des biens sociaux en fonction du statut personnel, mais il doit $s$ 'inspirer des pratiques 
dans la région de Baoding, la plus importante faisant travailler vingt mille personnes.

Désireux d'exploiter au mieux une telle source d'enrichissement, les villageois n'ont pas hésité au cours des dernières années à embaucher de la main-d'œuvre. Le tissage proprement dit est un travail exclusivement féminin : chacune des familles concernées de Baozi Pu emploie entre 4 et 22 jeunes filles extérieures au groupe domestique ${ }^{13}$; il s'agit de jeunes paysannes âgées de 16 ou 17 ans, originaires de régions plus pauvres de la province, et beaucoup viennent des villages où sont nées les épouses des paysans de Baozi $\mathrm{Pu}$. Si la population officielle du village était, en octobre 1986, de 704 personnes réparties au sein de 154 foyers (soit une moyenne de 4,6 individus par famille), la population totale réelle était en fait de 1500 individus, près de huit cents personnes enregistrées officiellement dans d'autres localités travaillant et vivant à Baozi Pu pour une durée indéterminée. Cette immigration subite explique en partie la vague récente de construction dans le village. Les nouveaux édifices obéissent à un plan fort simple : deux ailes rectangulaires viennent entourer l'ancienne habitation principale ; l'une fait office de dortoir pour le personnel salarié, l'autre accueille les métiers à tisser.

de gestion capitalistes... " (Cf. Social World Broadcast, 30 août 1989, FE/ 0548, B2/6). Il est probable qu'après juin 1989 les paysans ont continué de plus belle à investir leurs bénéfices dans des biens de consommation et non de production. Cette tendance était déjà dénoncée par les cadres locaux lors de notre séjour en octobre 1986 ; elle l'était encore lors de la visite d'une délégation de l'Académie des Sciences Sociales de Pékin. Il est possible également qu'il y ait eu une diminution des embauches extérieures.

13. Ces chiffres dépassent largement ceux prévus par la loi. Ainsi un ouvrage destiné aux paysans, intitulé Nongcun falü changshi wenda (Réponses à des questions courantes de droit rural) et publié en février 1985 par Falü chubanshe, indique (p. 126) : « Les familles qui possèdent une entreprise ou un commerce peuvent employer, lorsqu'elles sont confrontées à un problème technique particulier, 5 apprentis. Cette embauche doit faire l'objet d'un contrat spécifiant les devoirs et obligations des deux parties, ainsi que la durée de l'embauche et la rémunération perçue ». 
La production de tapis a pris une telle importance que l'avenir économique de la communauté est essentiellement lié à la prospérité de l'usine locale et à celle des ateliers domestiques qui travaillent pour elle. Les maisonnées qui ne possèdent pas de métier à tisser jouissent d'un revenu nettement inférieur aux autres. Au sein de notre échantillon, 18 familles travaillent en sous-traitance pour l'usine locale, les quatre autres ayant pour seules ressources les revenus tirés de la vente des produits agricoles et du travail salarié : le revenu annuel moyen des premières est de 6897 yuans, il n'est que de 2142 yuans pour les secondes.

Le développement de cette activité à la fois nouvelle et d'origine ancienne explique donc l'augmentation rapide du niveau de vie des habitants de Baozi Pu; mais la création de nombreuses entreprises et commerces au sein du district de Zuo a également été un facteur de croissance. Parmi les 104 membres des 22 foyers examinés, 40 possédaient en octobre 1986 un travail salarié, alors qu'on n'en comptait que 12 dans en 1978. 15 avaient été embauchés par l'usine de Baozi Pu et les 25 autres travaillaient à l'extérieur du village. Les salaires ainsi gagnés contribuent de façon importante à accroître le revenu familial. Ils s'échelonnent entre 30 et 180 yuans selon le métier exercé, et en 1986 représentaient en moyenne 387 yuans sur les 1230 gagnés par chaque individu de 1'échantillon.

Le développement de deux formes d'industrialisation fort différentes, l'une artisanale et familiale, l'autre collective et technologiquement plus sophistiquée, apparaît ainsi comme le fait marquant de la vie économique de Baozi Pu depuis le début des années quatre-vingt. C'est pourquoi il nous est apparu important de préciser le rôle attribué à chacune de ces deux formes et les relations qu'elles entretiennent entre elles, avant d'analyser comment le groupe domestique a répondu aux possibilités nouvelles qui s'offraient à lui. 


\section{Les industries domestiques et l'entreprise villageoise : une complémentarité réussie}

Les 18 familles de l'échantillon qui se consacrent au tissage artisanal de tapis possèdent en tout 48 métiers à tisser, soit 2,6 en moyenne par foyer ${ }^{14}$. Il convient de parler ici d'industries domestiques plutôt que d'entreprises privées. Le travail de production est en effet entièrement artisanal. Les métiers à tisser domestiques, en bois, valent 500 yuans, alors que le prix de ceux achetés par l'usine s'élève à 3000 yuans ${ }^{15}$. Ces petits ateliers ne possèdent par ailleurs aucun statut légal.

Quatre à cinq personnes peuvent travailler sur un même métier. C'est dès le premier entretien que nous avons eu la surprise de découvrir que le tissage était effectué par de la main-d'œuvre extérieure : les membres de la famille tissent parfois, mais ils opèrent surtout comme contremaîtres et préparent la laine, la trame, ainsi que les repas des employées.

Nos 18 familles emploient un total de 164 personnes se répartissant entre 158 ouvrières, 4 contremaîtres et 2 cuisiniers. Le phénomène majeur ainsi révélé, l'existence d'une main-d'œuvre immigrée exploitée par les propriétaires d'ateliers domestiques, aurait dû constituer un thème privilégié de l'enquête, mais il n'a pu faire l'objet d'une analyse détaillée : malgré nos tentatives pour modifier le questionnaire de façon à prendre en compte la situation imprévue qui s'offrait à nous, le malaise général régnant à propos de cette embauche extérieure nous a empêchés d'approfondir la question. « Il faut les considérer plutôt comme des parentes », nous assurait le chef du village. «Elles font partie de la famille. Elles ne mangent pas avec nous aujourd'hui parce que vous êtes là, sinon nous les traitons comme nos filles ! ", renchérissaient les paysans ${ }^{16}$. Nos tentatives pour parler avec les jeunes ouvrières étaient par ailleurs vouées à l'échec, un membre de la famille visitée venant aussitôt

14. Les familles de l'échantillon possèdent au minimum un métier et au maximum cinq.

15. Entretien, Baozi Pu, 9 octobre 1986.

16. Entretien, Baozi Pu, 8 octobre 1986. 
nous chercher pour nous montrer la dernière acquisition de la maisonnée en matière d'équipement électroménager.

La raison de ce malaise est apparue dès le premier repas passé ensemble lorsque l'un des paysans présents a lâché : « Certains nous accusent d'exploitation, mais en fait, les tisseuses gagnent bien plus d'argent que si elles étaient restées dans leur village... Plus elles travaillent, plus leur revenu augmente - et plus nous, c'est vrai, nous nous enrichissons ». Il se trouve en effet qu'un débat était alors en cours dans la presse chinoise, débat qui a duré jusqu'au mois de juin 1989, sur la façon dont il convient de considérer ce type d'embauche. (Depuis lors, le déclenchement d'une nouvelle campagne contre la « libéralisation bourgeoise », beaucoup plus menaçante que celle du printemps 1987, a réduit au silence les partisans de l'emploi de main d'œuvre salariée par des entrepreneurs privés.) Ainsi Mo Zhen, un chercheur cantonais, dénonçait-il, après avoir étudié les entreprises privées dans le district de Gaoyao, l'apparition d'une nouvelle classe d'exploiteurs : « 66 des 101 foyers enquêtés emploient 960 personnes, soit 14,5 personnes par famille en moyenne... Il existe une marge très importante entre les revenus de l'employeur et ceux de ses employés, ce qui élargit le fossé entre riches et pauvres. Dans certains cas, les employeurs sont totalement absents du travail de production ou de direction... et deviennent donc de nouveaux exploiteurs $»^{17}$. D'autres, comme Du Runsheng, se montraient plus modérés : « L'embauche de main-d'œuvre [par des industries privées]... possède une souplesse caractéristique des entreprises capitalistes, mais elle n'équivaut pas au capitalisme... Il y a matière à discuter sur le nombre de personnes que ces industries peuvent embaucher, mais même les membres du Parti doivent être autorisés à de telles pratiques ${ }^{18}$. Chacun reconnaissait cependant que le gouvernement était peut-être en mesure

17. Cf. Mo Zhen, « Tighten control over big labor-hiring households in rural areas m, Chinese economic studies, XXI, 2, 1987 (The private economy), pp. 90-99. Il s'agit de la traduction d'un texte chinois non publié.

18. Du Runsheng, «Du Runsheng tongzhi tan gugong wenti " (Le camarade Du Runsheng parle des problèmes d'embauche), Gongshang xinzheng guanli, 1986, 19. 
de poser certaines limites à ce genre de pratique, mais non d'y mettre un terme. Quoi qu'il en soit, les paysans que nous avons rencontrés craignaient fort de se voir accoler l'étiquette d'exploiteurs et essayaient de nier l'importance du phénomène d'embauche en établissant des liens de parenté, le plus souvent fictifs, avec les ouvrières.

C'est un fait qu'une grande souplesse caractérise les rapports entre les responsables des ateliers domestiques et les tisseuses. Les maisonnées ont le choix entre l'embauche d'apprenties ou d'ouvrières qualifiées. Les premières ne sont pas rémunérées pendant les six mois que dure leur formation, mais uniquement logées et nourries ${ }^{19}$. Elles accèdent ensuite au statut d'ouvrières qualifiées et perçoivent alors un salaire quotidien de 3,2 yuans, sur lequel le chef de famille prélève 1 yuan pour les frais de nourriture. Ce salaire correspond au tissage d'une pièce de 2 pouces (un peu moins de $5 \mathrm{~cm})$ de large et d'un pied $(30,48 \mathrm{~cm})$ de long.

Après avoir travaillé tous les jours pendant deux mois, les employées peuvent prendre un congé de cinq jours afin de rendre visite à leur famille. Comme on l'a vu, la plupart sont originaires de villages assez éloignés où les paysans de Baozi $\mathrm{Pu}$ possèdent sinon des parents, du moins des connaissances. Ce système est censé faciliter l'intégration des jeunes filles, mais il limite en fait leur indépendance. En effet, leur présence à Baozi Pu résulte dans la plupart des cas d'un accord conclu entre leurs parents et la famille qui les embauche, accord qu'il leur est difficile de remettre personnellement en cause. La durée de l'embauche n'est pas déterminée à l'avance mais dépend de la régularité et de l'ampleur des commandes. Lorsque celles-ci se font plus rares, ou lorsque les tapis commandés sont de petite taille, la main-d'œuvre excédentaire est renvoyée.

19. Le malaise manifesté par les paysans à propos de l'embauche de maind'œuvre extérieure s'est également exprimé sur ce point. Si, le premier jour, des chiffres vraisemblables nous étaient communiqués concernant le nombre d'ouvrières et d'apprenties employées par chaque foyer, dès le lendemain les paysans prétendaient n'employer que des ouvrières qualifiées, l'embauche d'apprenties leur paraissant plus susceptible d'être taxée d'exploitation. 
Seuls la préparation de la trame et le travail de tissage proprement dit sont effectués dans les ateliers privés. L'usine effectue plutôt dans ce domaine un travail de formation des ouvrières, lesquelles retournent ensuite dans leur village natal pour y créer de nouveaux ateliers domestiques. Elle joue par contre un rôle déterminant lors des différentes phases du travail de fabrication situées en amont et en aval du tissage. La laine y est traitée de façon industrielle. La réalisation des motifs, le choix des couleurs relèvent de la responsabilité de techniciens spécialisés qui travaillent en fonction des demandes exprimées par le département du commerce extérieur de la province du Hebei, lequel est plus au fait du marché d'exportation. De même, le contrôle de la qualité du produit fini, le lavage, le séchage et l'étirage des tapis - activités pour lesquelles l'usine a fait l'achat d'équipements importants - sont accomplis de façon industrielle.

L'usine vend les tapis au département du commerce extérieur de la province du Hebei au prix de 62 yuans pour une pièce de 1 pied carré (soit de $30,48 \mathrm{~cm}$ de côté). $24 \%$ de cette somme seraient destinés à l'achat de la laine, et $13 \%$ couvriraient les frais de gestion de l'usine, les salaires, les impôts, l'autofinancement des investissements, les dépenses sociales... Les $63 \%$ restants, soit 39,06 yuans, sont remis aux familles paysannes. Or il faut cinq jours à une ouvrière pour tisser une pièce d'un pied carré. Elle perçoit donc 16 yuans sur les 39,06 payés par l'usine à son patron, soit un peu plus de $40 \%$.

Au dire des paysans, les commandes sont aléatoires, et les ateliers privés sont loin de fonctionner toute l'année. Un métier à tisser rapporterait ainsi, selon eux, entre 3000 et 4500 yuans par an à son propriétaire, selon la rapidité d'exécution des ouvrières, la durée des périodes d'activité, etc... Il plus que probable, cependant, que les paysans dissimulent une certaine fraction de leurs revenus.

Les bénéfices réalisés par l'usine villageoise ne sont pas directement redistribués aux familles : ils sont largement réinvestis dans la production. Ils permettent également de financer un fonds collectif destiné au paiement des impôts agricoles et à la prise en charge de différents services sociaux. C'est l'usine, par exemple, qui procède aux dépenses nécessaires pour assurer le bon fonctionnement de l'école pour adultes du 
village. C'est elle également qui verse un revenu minimum aux paysans âgés dépourvus de moyens de subsistance. De plus, un système d'assurance sociale a été mis en place qui permet aux paysans d'être remboursés en partie des frais de maladie. Enfin, dans le domaine de l'éducation, les responsables de l'usine récompensent les parents qui incitent leurs enfants à poursuivre des études. Ainsi, lorsque les résultats scolaires dépassent la note de 70 sur 100 , les parents reçoivent 50 yuans pour un élève de l'école primaire, 80 yuans pour un élève de l'école secondaire et 300 yuans pour un étudiant à l'université.

Outre le pouvoir qu'ils détiennent dans le domaine économique, les cadres de l'entreprise villageoise exercent donc une influence politique et sociale déterminante. Ils président les réunions fréquentes du Parti et maintiennent des « organisations de masse » comme la milice ou la Ligue de la jeunesse communiste, qui ont souvent disparu dans d'autres villages ${ }^{20}$. Les dirigeants de l'usine sont en fait les mêmes que ceux du village, et Baozi Pu apparaîtra de ce fait, selon le point de vue adopté, comme un village au sein duquel s'est développée une entreprise collective ou comme une usine possédant le droit d'exploiter quelques centaines de mou destinés à la consommation locale.

Nous avons vu que ce sont les cadres de la brigade de production qui avaient pris en 1980 l'initiative de remettre à l'honneur la fabrication de tapis. Depuis, aucune élection n'a eu lieu pour désigner des nouveaux responsables. Un comité aurait dû être élu par l'ensemble de la population après la disparition des communes populaires, mais les dirigeants en place n'ont jamais convoqué de réunion en ce sens et les paysans, peu désireux de heurter ceux qui leur avaient permis de connaître une prospérité aussi rapide, n'ont pas réagi. L'ancien vice-secrétaire de la cellule du Parti de la brigade, qui dirige l'usine, est le principal respon-

20. En août 1986, dans un village de la région de Jiujiang, dans le district de Nanhai (Guangdong) où nous avons eu l'occasion de nous rendre, la Ligue de la jeunesse communiste avait cessé toute activité, la milice avait été remplacée par un petit groupe chargé de la sécurité, et aucun membre du Parti n'avait été nommé depuis 1982. La même situation prévalait dans un village du district de Taishan (Guangdong). 
sable du comité villageois. Le secrétaire de la cellule du Parti, second cadre de l'usine, est chargé des affaires liées à la sécurité. Les problèmes de santé et d'hygiène ont été confiés à uh troisième responsable de l'entreprise. Ainsi, contrairement à la situation en vigueur dans la plupart des communautés chinoises, où les membres des comités villageois n'exercent pas de fonctions déterminantes dans le domaine de la production, les responsables de Baozi Pu contrôlent étroitement la vie économique locale.

Mais si la réussite de Baozi Pu s'explique en partie par les talents d'entrepreneurs de ses dirigeants, elle résulte également de la complémentarité instaurée entre l'usine et les petits ateliers privés. Sans la présence de la première, les industries domestiques n'auraient pas connu d'essor aussi rapide. A l'inverse, l'usine n'aurait pas bénéficié d'une telle croissance sans la création des ateliers. Il lui aurait été impossible par exemple d'embaucher aussi rapidement près de 3000 personnes. Les habitants de Baozi Pu sont parfaitement conscients de ce rapport de complémentarité, qu'ils expriment à l'aide de la formule suivante: «Elle est la tête du dragon et nous sommes la queue du phénix ! " (longtou fengwei $)^{21}$.

Cette complémentarité n'a rien d'exceptionnel. De telles situations ont été fréquentes pendant les premières phases du développement industriel en Europe et en Amérique du Nord. Citons ici les propos de Thomas Dublin, qui conclut ainsi son analyse de l'économie de la Nouvelle Angleterre entre 1830 et 1850 : « Le travail à l'usine et les activités productives de sous-traitance effectuées en milieu domestique pouvaient constituer deux aspects du travail de production pour le marché se complétant et se renforçant mutuellement ${ }^{22}$.

Ces deux systèmes de production, l'un domestique et artisanal, l'autre industriel et collectif, ont influencé de façon parfois contradictoire les relations au sein du groupe familial en matière de division du travail et d'économie domestique, et c'est ce que nous allons étudier à présent.

21. Entretien, Baozi Pu, 10 octobre 1986.

22. Cf. Thomas Dublin, «Women's work and the family economy : textiles and palm leaf hatmaking in New England, 1830-1850 », La revue Tocqueville, V, 2, 1983, pp. 297-316. 


\section{Les nouvelles stratégies familiales en matière de division du travail}

La distribution des différentes activités de production au sein du groupe domestique et la répartition du pouvoir de décision économique nous sont connues grâce aux entretiens menés avec les 22 familles étudiées et aux questionnaires qu'elles ont accepté de remplir.

L'échantillon se répartit en 15 familles nucléaires et 7 familles souches ${ }^{23}$. Dans le cas de ces dernières, 4 sont des familles souches au sein desquelles deux couples sont présents, alors que les 3 autres ne regroupent que la mère, l'un de ses enfants mariés, son conjoint et les petitsenfants $^{24}$. Comme on l'a vu, ces différents groupes domestiques rassemblent 104 personnes, 53 de sexe féminin et 51 de sexe masculin. L'âge moyen des femmes s'élève à 33,6 ans et celui des hommes à 27,6 ans. La coutume qui veut qu'un homme épouse volontiers une femme de quelques années son aînée demeure très répandue dans la région. Dans 10 des 26 couples de l'échantillon, l'épouse est plus âgée que son mari, de 2,6 ans en moyenne ; dans 13 autres couples, c'est l'époux qui est l'aîné, de 3,7 ans en moyenne ; aucune différence d'âge ne sépare l'homme et la femme dans les trois derniers cas.

L'analyse du niveau d'instruction révèle une progression très nette au cours des trois dernières décennies, surtout en ce qui concerne la population féminine. Au sein de la tranche d'âge née avant 1932, c'est-à-dire ayant achevé ses études avant l'arrivée du Parti communiste au pouvoir, les hommes ont été scolarisés pendant six ans en moyenne et les femmes

23. Les trois types fondamentaux de groupes familiaux sont la famille conjugale ou nucléaire, qui regroupe le père, la mère et les enfants non mariés; la famille souche, caractérisée par la présence de plusieurs couples vivant sous le même toit, mais avec un seul couple à chaque génération; et la famille étendue ou indivise, qui comprend les grands-parents, leurs enfants mariés, des petits enfants et éventuellement des arrière petits-enfants.

24. Dans l'une de ces trois familles, c'est la mère de l'épouse et non celle de l'époux qui est présente. Cette distinction est importante dans la mesure où, comme nous le verrons, la famille souche composée d'une mère, de sa fille, de son gendre et de ses petits-enfants fonctionne en réalité comme une famille nucléaire, la belle-mère ne possédant aucun droit particulier. 
pendant un an et trois mois. Dans la tranche née entre 1932 et 1943 (ceux qui ont pu faire une partie de leurs études après 1949), les hommes ont été à l'école pendant 8,2 ans et les femmes pendant 4,2 ans. Enfin, les chiffres sont respectivement de 8,7 ans et de 8,1 ans pour les individus nés après 1943 (dont la scolarité a donc débuté au plus tôt en 1950) ayant aujourd'hui achevé leurs études.

Les activités professionnelles des 104 individus de l'échantillon sont très variées, comme le montre le tableau ci-dessous :

Tableau 1

Activité professionnelle des membres de l'échantillon ${ }^{25}$

\begin{tabular}{|c|c|c|c|}
\hline & nes & Femmes & Total \\
\hline Jeunes enfants & 5 & 3 & 8 \\
\hline Élèves et lycéens & 12 & 9 & 21 \\
\hline Ouvriers, techniciens & 16 & 7 & 23 \\
\hline Cadres industriels & 5 & 1 & 6 \\
\hline Employés secteur tertiaire & & & \\
\hline hors village & 5 & 3 & 8 \\
\hline Salariés jardin potager collectif & 2 & - & 2 \\
\hline Soldat & 1 & - & 1 \\
\hline Commerçants privés & 2 & - & 2 \\
\hline Fabrication domestique de tapis & 2 & 16 & 18 \\
\hline Travaux domestiques & - & 11 & 11 \\
\hline Inactifs & 1 & 3 & 4 \\
\hline TOTAL & 51 & 53 & 104 \\
\hline
\end{tabular}

25. Si l'activité agricole n' apparaît pas, c'est parce que personne ne s'y consacre exclusivement de façon quotidienne. Comme on verra, ce sont en général les femmes qui sont chargées de l'entretien des parcelles cultivées, en plus des travaux domestiques et du contrôle de la fabrication des tapis. 
On voit donc à quel point les activités subsidiaires comme le travail salarié dans les entreprises ou les commerces locaux occupent désormais une place prépondérante. Cette situation reflète l'acquisition par les familles paysannes d'une certaine autonomie sur le plan de la division du travail.

La redistribution des terres aux maisonnées et l'établissement de forfaits d'exploitation conclus directement avec les groupes domestiques ont libéré la main-d'œuvre paysanne. On est loin de la situation prévalant sous le système collectiviste, dans laquelle les tâches collectives étaient distribuées une ou deux fois par jour à une population active souvent trop abondante. L'organisation de grands travaux (construction de réservoirs, entretien des routes), parfois utiles mais aussi destinés à occuper une force productive désœuvrée, a cessé. La maisonnée a retrouvé ses fonctions d'unité de production de base, et c'est ce qui a permis l'essor des petites industries domestiques du type de celles de Baozi Pu.

Différentes stratégies familiales de division du travail ont surgi qui obéissent toutes à un même principe : utiliser au mieux les ressources du groupe domestique. Or, ces ressources, vu la faible superficie des terres disponibles, c'est essentiellement la main-d'œuvre familiale. L'exploitation aussi rationnelle que possible - même si elle reste influencée par des critères culturels - de cette main-d'œuvre apparaît ainsi comme le souci dominant des chefs de famille.

Deux grands types d'activité peuvent être ici distingués : les activités de production se déroulant au sein de la maisonnée, et celles qui s'accomplissent à l'extérieur.

\section{Une division du travail au service des intérêts du groupe}

Quelle que soit l'activité professionnelle de chacun, tous les membres du foyer âgés de 10 à 65 ans environ participent de façon plus ou moins intense au travail de production domestique. Les femmes les plus âgées se chargent de nourrir la basse-cour et de préparer la pâtée destinée aux cochons. Les plus jeunes, une fois de retour de l'école, collaborent à l'entretien des parcelles et sont présents lors des gros travaux agricoles, semailles et récoltes. 
Ce sont bien entendu les femmes qui s'occupent des tâches ménagères. Dans 20 des 22 familles étudiées, c'est à la femme la plus âgée que revient le soin de répartir chaque jour les travaux domestiques entre celles qui n'ont pas d'emploi à l'extérieur. Dans un cas, c'est la fille, âgée de 23 ans, qui assume seule l'ensemble des travaux ménagers; et au sein de la dernière famille, les travaux domestiques sont distribués de façon informelle.

Bien souvent la main-d'cuuve féminine est également chargée des travaux agricoles quotidiens (déposer de l'engrais, arracher les mauvaises herbes, etc.) : 17 familles citent une femme comme principal responsable, les hommes n'étant mentionnés que cinq fois ${ }^{26}$. Outre l'embauche d'une partie des hommes actifs à l'extérieur, la petite taille des parcelles attribuées et la nature des produits cultivés expliquent la prépondérance des femmes dans le travail agricole. Les céréales réclament des soins quotidiens moins intensifs que les cultures potagères : or la plupart des familles produisent du blé, du maïs ou du millet et achètent leurs légumes à la collectivité.

Une maisonnée ne peut envisager d'acheter de métier à tisser et de créer d'atelier domestique que si l'un au moins de ses membres (une femme dans la plupart des cas) maîtrise parfaitement la technique et peut diriger les ouvrières. Tel est bien l'obstacle qui empêche trois des 22 familles étudiées de se lancer dans cette activité. Dans deux d'entre elles il n'y a d'autre main-d'œuvre féminine disponible qu'une paysanne âgée (63 et 70 ans respectivement), qui ne sait pas tisser. Dans la troisième, la mère, âgée de 43 ans, est cadre à l'usine villageoise mais ignore tout de l'art du tissage. Le cas d'une quatrième famille, également sans activité tapissière, est différent : il s'agit du foyer d'un ancien cadre de la brigade qui entretient de très mauvaises relations avec les responsables actuels.

26. Différents membres de la maisonnée peuvent toutefois se charger de ce travail, qu'ils assument alors à tour de rôle et selon leur disponibilité. Dans l'une des familles étudiées le père, chauffeur, est fréquemment absent du village : c'est donc la mère, employée à l'usine du village, qui le soir venu apporte aux champs les soins requis, aidée par ses deux filles âgées de 12 et 14 ans. Le troisième enfant, un fils âgé de 10 ans, est jugé trop petit pour se joindre à elles. 
Les femmes jouent un rôle majeur au sein de l'atelier domestique, que ce soit au niveau du tissage, de la préparation de la trame et de la laine, de la surveillance des ouvrières, ou de la cuisine. Si, dans l'échantillon, 16 femmes et 2 hommes ont pour principale responsabilité de diriger quotidiennement le travail de tissage, 39 personnes au total participent à la bonne marche de l'industrie domestique, dont 27 femmes.

Les différents membres du groupe domestique ne sont pas libres de décider eux-mêmes de leur fonction au sein de l'atelier : c'est en général le chef de famille qui attribue à chacun son rôle. Dans 13 des 18 familles concernées il en a décidé seul, alors que dans les cinq autres il a consulté son conjoint et le reste du groupe.

Plus le nombre de métiers à tisser est élevé, plus la coopération entre les membres de la famille est nécessaire. Les quelques exemples qui suivent illustrent un certain nombre de combinaisons. L'une des familles étudiées est composée du mari, chauffeur, de son épouse, cadre à l'usine villageoise, et de leurs trois enfants qui poursuivent leurs études scolaires. Chacun des deux époux ayant un emploi salarié, la famille ne possède qu'un métier à tisser et a embauché quatre ouvrières. La femme prépare le soir les écheveaux de laine, trace tous les matins sur la trame le trait qui indique le travail à effectuer dans la journée, et vient à l'heure du déjeuner vérifier la qualité du tissage.

Dans une autre famille, qui regroupe six personnes, ce sont la mère (64 ans) et la belle-fille ( 26 ans) qui s'occupent de tout ce qui concerne le tissage, la première étant surtout chargée de préparer la nourriture des douze ouvrières embauchées, alors que la seconde joue le rôle de contremaître et aide les employées à résoudre les difficultés techniques qui peuvent surgir.

Dans un autre exemple, le mari, âgé de 45 ans, est établi à son compte comme meunier et dirige le travail de douze tisseuses. La cuisine et la préparation de la laine incombent à son épouse, également chargée des travaux domestiques.

Si les femmes jouent, comme on l'a vu, un rôle déterminant dans le processus - s'agissant d'une technique traditionnelle qu'elles sont les premières à maîtriser, et considérant que la majorité d'entre elles étaient appelées de toute façon à rester au foyer - c'est au chef de famille, en 
général un homme, qu'il revient de vérifier le soir venu la qualité du travail accompli, et surtout celle d'aller vendre le produit fini à l'usine. En effet les contacts entre les ateliers familiaux et l'usine locale se doivent de passer par l'intermédiaire du membre de la maisonnée possédant le plus grand prestige social, et c'est par conséquent un homme, dans 15 familles sur 18, qui est chargé d'aller remettre les tapis à l'usine et de négocier les commandes suivantes.

Cette entrevue revêt une importance capitale dans la mesure où les cadres y déterminent si les produits livrés sont de première ou de seconde qualité, ce qui bien sûr modifie de façon sensible le prix d'achat. Il ne faut pas oublier non plus qu'aucun quota de production n'est assigné aux industries domestiques et que les commandes sont aléatoires. Leur régularité, la commande de petits ou de grands formats ont, comme nous l'avons déjà vu, des conséquences non négligeables sur les ressources annuelles de la maisonnée.

Parmi les trois femmes se chargeant des relations avec l'usine, l'une y travaille précisément comme technicienne et les relations privilégiées qu'elle entretient avec les responsables locaux la désignent de façon automatique comme représentante du groupe familial. Dans le cas des deux autres, en revanche, ce sont leurs époux qui exercent des fonctions de cadre dans l'entreprise, et qui en raison du pouvoir qu'ils y détiennent ne sauraient aller eux-mêmes vendre les tapis fabriqués par leur famille sans courir le risque d'être accusés de tirer profit de leur situation.

Ainsi, les principes traditionnels de partage des responsabilités entre hommes et femmes demeurent relativement inchangés au sein des industries domestiques : quelle que soit l'importance du rôle exercé par les secondes dans le domaine de la production, les contacts sociaux importants s'accomplissent plus volontiers par l'intermédiaire des premiers.

De même, le principe de l'autorité des générations supérieures est largement respecté. Dans 16 familles sur 18 c'est à elles qu'appartient la personne (homme ou femme) qui a pour mission de vendre la production domestique de tapis. Les deux exceptions sont deux jeunes ouvriers célibataires, âgés respectivement de 21 et de 26 ans, employés à l'usine du village, qui pour cette raison ont été choisis comme représentants du groupe familial auprès d'elle. 
Vers une diversification croissante des activités de production

Les chefs de famille n'en ont pas moins le souci de diversifier le plus possible leurs activités. Les paysans ignorent en effet combien de temps se maintiendra l'orientation politique actuelle, et ils préfèrent combiner le développement du tissage domestique de tapis avec l'embauche d'une partie de la main-d'œuvre familiale dans les usines locales.

Quarante personnes (29 hommes et 11 femmes) exercent ainsi une activité professionnelle à l'extérieur du groupe domestique, dont quinze (10 hommes et 5 femmes) sont employées par l'usine villageoise. Vu l'importance acquise par celle-ci au cours des dernières années, c'est autant que possible le chef de famille qui va y travailler, de telle sorte que soient facilités les rapports économiques et sociaux du groupe domestique avec les cadres locaux. Il existe une autre raison à la prépondérance des hommes (outre leur aptitude présumée au travail en usine) : placer une de ses filles dans l'entreprise de Baozi Pu est considéré comme peu habile puisque la famille ne pourra jouir du revenu ainsi gagné que jusqu'au mariage de la jeune femme, laquelle ira alors vivre chez ses beauxparents, dans un village souvent trop éloigné pour qu'elle puisse envisager de conserver son emploi. Le même calcul explique pourquoi, face à une proposition de travail salarié réservée uniquement aux femmes, les paysans estiment que c'est à la mère de profiter de l'opportunité (14 réponses sur 22), et sinon, à la fille célibataire la plus jeune (6 réponses), donc à celle qui restera le plus longtemps sous le toit familial.

Si les membres des générations supérieures s'efforcent de maintenir leur autorité dans le domaine de la division du travail, s'ils peuvent assigner à chacun une fonction déterminée au sein de l'atelier domestique et essayer de peser sur le choix de celui ou de celle qui acceptera une proposition de travail salarié, leur pouvoir dans ce dernier domaine n'est pas illimité. En effet les propositions de travail ne leur sont pas toujours directement adressées. Les membres des jeunes générations sont donc de plus en plus nombreux à posséder un emploi salarié, ce qui accroît leur indépendance.

Vingt personnes, parmi les quarante de notre échantillon travaillant à l'extérieur de la maisonnée, ont moins de trente ans. Parmi les sept 
hommes célibataires, on compte deux chauffeurs, trois ouvriers, un technicien et un étudiant. Les activités professionnelles des douze jeunes femmes célibataires recensées se répartissent de la façon suivante : cinq travaillent dans les ateliers domestiques, cinq sont ouvrières, une est technicienne, et la dernière est médecin. Il est difficile d'extrapoler à partir d'un échantillon aussi restreint, mais il semble que les femmes aient tendance à retarder l'âge de leur mariage afin de bénéficier plus longtemps de l'aisance qui caractérise les foyers de Baozi Pu (certaines d'entre elles ont 28 ou 30 ans). Les hommes par contre se marient plus tôt, et dans le cas de tous les mariages conclus depuis 1980 la belle-fille travaille dans l'atelier domestique de tissage.

Les réformes économiques mises en oeuvre depuis le début des années quatre-vingt ont donc modifié de façon considérable l'utilisation de la main-d'œuvre à Baozi Pu. Les activités de production ont été diversifiées en fonction du nombre de travailleurs actifs au sein de la famille. Le principe selon lequel les hommes se chargent des activités de production tournées vers la société et les femmes de celles qui sont liées à la maisonnée conserve une certaine influence ${ }^{27}$, mais il n'est plus exclusif. Enfin, le maintien d'une autorité parentale forte caractérise la répartition des activités de production. Ces stratégies de division du travail, faites de changement et de continuité et soutenues par une gestion du budget familial fort traditionnelle, expliquent en partie l'enrichissement rapide des foyers de Baozi Pu.

\section{Les normes de répartition des ressources familiales}

Une accumulation favorisée par le principe de la communauté de budget

La distinction posée plus haut entre deux grands types d'activités professionnelles, celles qui sont accomplies à l'intérieur de la maisonnée et celles qui s'exercent à l'extérieur, correspond également à deux modes de

27. Dix de nos interlocuteurs ont d'ailleurs invoqué au cours des entretiens l'expression nan wai nü nei (les hommes à l'extérieur, les femmes à l'intérieur). 
rémunération. Aucun salaire n'est en effet attribué aux membres de la famille qui participent d'une façon ou d'une autre à la production de tapis : le revenu tiré de la vente des tapis est versé à celui ou à ceux qui détiennent l'autorité en matière budgétaire. Dans une seule des dix-huit familles de l'échantillon la mère remet au début de l'année quelques dizaines de yuans à sa fille, laquelle, âgée de 18 ans et célibataire, surveille le travail quotidien des ouvrières. (Elle n'en doit pas moins obtenir l'accord de sa mère avant de procéder à une quelconque dépense). On peut en dire de même pour les ressources provenant de la vente des produits agricoles. En d'autres termes, la population féminine dont l'activité de production se déroule à l'intérieur de la maison n'est pas rémunérée directement, même si elle effectue un travail marchand. Cette absence de rémunération est une caractéristique fréquente des économies domestiques où les femmes et les enfants sont voués à des activités d'artisanat ${ }^{28}$.

De même, la majorité de ceux qui possèdent un emploi salarié - mais pas tous, on va le voir - remettent la totalité de leur revenu au groupe familial. La notion de revenu individuel n'existe donc pas. Même s'ils sont gérés par un ou deux membres de la maisonnée, capitaux et biens appartiennent à la collectivité.

C'est avec les nouveaux droits octroyés dans certains cas aux salariés que la prépondérance du groupe domestique trouve ses limites. Parmi les quarante personnes de l'échantillon exerçant un emploi salarié, dix conservent une partie de leur salaire, dans une proportion qui varie entre 20 et $50 \%$. Il s'agit tout d'abord de deux hommes mariés travaillant l'un à Pékin et l'autre dans la province du Shanxi, qui prélèvent quelques dizaines de yuans sur leur revenu mensuel afin de pourvoir à leurs dépenses quotidiennes. Quatre des sept jeunes filles et quatre des cinq paysans célibataires qui travaillent à l'extérieur de la maisonnée ne remettent également au chef de famille qu'une partie de leur salaire - la

28. Thomas Dublin, dans l'étude citée plus haut, remarque : « Pour les familles de Fitzwilliam, l'activité de production de chapeaux des femmes mariées était souvent considérée comme faisant partie intégrante d'une économie domestique contrôlée par le mari ». Cf. Dublin, art. cité, p. 301. 
majeure partie, il est vrai. Aux yeux des habitants de Baozi Pu cette tolérance se justifie par le fait qu'il s'agit de jeunes gens ayant acquis un statut social plus élevé depuis qu'ils ont quitté la condition paysanne. Les contacts plus étroits qu'ils entretiennent avec la société extérieure, la recherche d'un éventuel conjoint parmi leurs collègues, les obligent à effectuer certaines dépenses de prestige. Ainsi un paysan explique-t-il : «C'est une question de face. Nos enfants qui travaillent à l'usine ont beaucoup d'amis. Ils doivent pouvoir offrir de temps en temps un repas au restaurant, aller au cinéma, s'habiller comme les jeunes de la ville... ${ }^{29}$. Un seul des quarante salariés de l'échantillon conserve la totalité de son salaire : il s'agit d'un ouvrier marié, âgé de 23 ans, dont le revenu mensuel sert aux dépenses de la petite famille qu'il forme avec sa femme et leur nouveau-né, bien qu'ils vivent sous le même toit que ses parents et qu'il n'y ait pas eu de division familiale.

Il y a donc une inégalité certaine entre les jeunes célibataires employés à l'extérieur de la famille et ceux dont la principale activité de production se déroule au sein de la maisonnée. La famille est amenée à faire des concessions aux premiers puisqu'elle ne peut plus prétendre contrôler entièrement leur revenu, qui leur est versé individuellement. On parlera à leur propos d'augmentation de leur « pouvoir de marchandage $\gg^{30}$.

Cela dit, 29 d'entre les 40 salariés de l'échantillon n'en remettent pas moins la totalité de leurs gains au groupe domestique. Celui-ci fonctionne donc bien comme une unité d'épargne, puisque le chef de famille contrôle non seulement les profits qui lui reviennent directement (ceux de la vente des tapis et des récoltes), mais prélève aussi la plus grande partie des salaires individuels. Les sommes ainsi réunies sont importantes. Elles permettent l'acquisition rapide de biens de production et de consommation, d'autant mieux que le pouvoir de décision en matière de dépense est le plus souvent monopolisé par une ou deux personnes.

29. Entretien, Baozi Pu, 11 octobre 1986.

30. Sur ce concept voir Nancy Folber, « Household economic analysis : a nonneoclassical approach », Economic development and cultural change, 32, 2 (1984), pp. 303-330. 


\section{Le maintien d'une distribution du pouvoir verticale}

Le chef de famille joue un rôle important dans l'organisation des différentes activités de production, on l'a vu ; son rôle dans la distribution des ressources est plus important encore. Le plus souvent c'est à lui que sont remis les différents revenus générés par le groupe domestique, ou à lui et à son conjoint : dans ce dernier cas, l'argent est déposé dans un tiroir auquel le couple a librement accès. Neuf familles au total ont adopté l'usage du tiroir commun (huit familles nucléaires et une famille souche). Dans cinq autres cas (quatre familles nucléaires et une famille souche) c'est l'homme le plus âgé qui contrôle les ressources familiales, et la femme la plus âgée dans six autres cas (trois familles nucléaires et trois familles souches). Une belle-fille âgée de 53 ans tient les cordons de la bourse au sein d'une autre famille souche, et une dernière famille n'a pas accepté de répondre à la question.

Le pouvoir semble moins souvent concentré entre les mains d'une seule personne au sein des familles nucléaires. La structure sociale plus complexe des familles souches exige-t-elle une autorité plus définie ? C'est en effet à l'homme de la génération supérieure, ou à son épouse, que sont remis les revenus tirés des différentes activités de production. Dans l'une des sept familles souches étudiées, qui regroupe une paysanne âgée de 63 ans, sa fille, son gendre et ses petits-enfants, c'est le gendre qui assume l'entier contrôle du budget familial, ce qui illustre la position inférieure occupée par la mère de l'épouse au sein de la maisonnée.

L'analyse de la distribution du pouvoir de décision en matière d'achat de biens d'équipement ou de consommation importants confirme la faible influence accordée aux membres des générations inférieures. La décision revient à l'homme le plus âgé dans huit cas (cinq familles nucléaires et trois familles souches), à la femme la plus âgée dans six cas (cinq familles nucléaires et une famille souche), au couple de parents au sein de trois familles nucléaires, et enfin à la belle-fille dans une famille souche. Deux familles (l'une souche et l'autre nucléaire) ont choisi la concertation entre les membres du groupe domestique, et deux n'ont pas répondu à la question. 
Prenons l'exemple de deux familles. La première est composée du mari (41 ans), technicien dans une usine à l'extérieur du village, de son épouse (39 ans), qui s'occupe de l'atelier domestique, et de leurs trois enfants âgés respectivement de 15,11 et 4 ans. Les différents revenus familiaux sont remis au mari, mais les décisions importantes en matière d'achat sont prises par le couple.

La seconde regroupe la mère (67 ans), qui s'occupe des tâches ménagères tout en participant aux activités liées à la fabrication de tapis, son fils (47 ans), comptable à l'usine villageoise, sa belle-fille (40 ans), qui prépare la nourriture des 18 employées, son petit-fils ( $20 \mathrm{ans}$ ), technicien dans une usine des environs, l'épouse de son petit-fils ( 21 ans), qui contrôle le tissage des tapis, sa petite-fille (18 ans), ouvrière, et enfin un autre petit-fils (18 ans aussi), qui poursuit ses études. Les différents revenus, salaires compris, sont remis en totalité à la belle-mère, qui décide seule des dépenses familiales.

Le pouvoir de décision, quand il s'agit de l'acquisition de biens tels que machine à laver, télévision, réfrigérateur ou tracteur, de la construction d'une maison, etc., est donc réservé dans la plupart des cas à un seul individu (15 réponses sur 22). En dépit d'une légère prédominance masculine, les femmes jouent un rôle important puisque ce pouvoir leur revient au sein de 7 familles. Il s'agit souvent de paysannes dont l'époux est décédé, ou absent parce que son travail le retient dans une autre localité. Dans trois cas, cependant, la femme tient les cordons de la bourse bien que son époux soit présent à la maison. Il n'en faut pas moins souligner que cette situation ne constitue pas un changement très significatif dans la mesure où, dans les décennies précédentes déjà, les femmes étaient souvent en charge du budget familial. Et surtout cela ne remet pas en cause le principe selon lequel les hommes sont les principaux responsables des contacts avec la société $e^{31}$.

31. La persistance de la distinction nan wai nü nei apparaît par exemple lor squ'on analyse les modes de résolution des conflits : les hommes interviennent dans les cas de conflits mettant en cause des membres de la famille qui ont également des contacts étroits avec la société, alors que les femmes exercent plutôt leur autorité sur ceux qui vivent et travaillent au sein du groupe domestique. C'est la mère qui intervient la plupart du temps lorsqu'une dispute 
Si une certaine égalité apparaît donc entre les sexes au niveau de la gestion du budget, la structure hiérarchique familiale demeure largement déterminée par l'âge. Dans deux familles seulement tous les membres, quel que soit leur âge, leur sexe et leur situation maritale, sont invités à exprimer leur point de vue sur l'utilisation des ressources familiales. D'une façon générale, les membres des générations inférieures doivent, s'ils désirent procéder à des dépenses personnelles, obtenir au préalable l'accord du chef de famille, qui leur remet ensuite la somme nécessaire. Les belles-filles notamment, qui jouent le rôle de contremaître dans les ateliers de tissage, n'ont pas de revenu individuel et sont obligées de s'adresser à leur belle-mère en cas de besoin.

Cette autorité du chef de famille, et en général des membres les plus âgés du groupe domestique, demeure également très étendue lors des grands événements sociaux qui marquent la vie familiale, par exemple les mariages. Il est fondamental de choisir une belle-fille qui sache se soumettre aux impératifs de la maisonnée et prendre en charge certaines responsabilités au sein de l'entreprise domestique. Si l'unité du groupe se trouve menacée par la différenciation de ses activités économiques, les parents s'efforcent en revanche de consolider leur pouvoir en intervenant de façon autoritaire dans le choix de leur futur gendre et surtout de leur future bru. Ainsi les représentants de dix-huit familles dans l'échantillon

éclate entre ses jeunes enfants et ceux d'autres maisonnées (21 cas sur 22). C'est la belle-mère qui s'interpose en cas de querelle entre sa belle-fille et d'autres membres du village (20 cas sur 22) : le mari ne se manifeste en général pas car les époux ne doivent pas exprimer de façon trop ouverte les liens qui les unissent. Par contre, lorsqu'un fils majeur est mêlé à un conflit social, c'est le père qui intervient de préférence ( 18 cas sur 22 ), à moins qu'il ne travaille dans une autre localité. Enfin, c'est au mari d'intervenir pour apaiser une dispute opposant sa femme à d'autres paysannes, si du moins ils ont tous deux atteint un certain âge et ne vivent plus sous le même toit que leurs parents. En revanche les femmes sont impuissantes quand un différend éclate entre leur époux et d'autres villageois : ce sont alors les membres du comité de conciliation du village, trois anciens cadres à la retraite, qui proposent leurs services de médiateurs. Ils ne sont cependant habilités qu'à régler les conflits entre maisonnées, et non ceux qui peuvent surgir au sein même d'un groupe familial. 
affirment-ils que les enfants doivent absolument obtenir l'accord de leurs parents sur le choix de leur futur conjoint. Quatre interlocuteurs seulement indiquent que ce n'est pas vraiment nécessaire.

Outre le poids des normes sociales, une autre raison explique sans doute que chacun semble aujourd'hui encore s'accommoder d'une structure hiérarchique familiale verticale et relativement rigide. Tous en effet tirent profit du tissage domestique de tapis, et il est probable que les sacrifices consentis individuellement sur le plan financier sont compensés par le niveau de vie élevé que garantit la cohésion du groupe.

\section{Conclusion}

L'analyse de l'économic domestique des habitants de Baozi Pu met en valeur deux phénomènes importants : la diversification, aussi poussée que possible, des activités de production des membres de la famille, et le maintien d'une structure hiérarchique rigide qui oriente la division du travail ainsi que la distribution des richesses.

La diversification rationnelle des activités de production permet d'augmenter le revenu familial, et la cohésion du groupe domestique, consolidée par l'existence d'une autorité centrale bien définie, offre à la maisonnée la possibilité de procéder à une épargne et à des investissements rapides.

Que la diversification des activités professionnelles soit l'un des modes d'enrichissement possibles est souligné par différentes études chinoises. La revue Lilun jiaoyu de Taiyuan insiste par exemple, dans sa livraison de janvier 1988, sur la nécessité pour les familles paysannes qui désirent s'enrichir d' « organiser la main-d'œuvre de façon à multiplier les activités »(zuzhi laodong jilei huodong) $)^{32}$. Or, plus le nombre de membres actifs au sein du foyer est élevé, plus une telle diversification

32. Cf. Li Shuiping, « Jiating jilei shi shixing nongye jieyue jingying de guanjian jiegou " ( $L$ 'accumulation familiale est le moyen fondamental de procéder à une gestion économique de l'agriculture), Lilun jiaoyu, 1988, 1, pp. 155-156. 
est possible. Rien de surprenant, de ce fait, si les études portant sur les " foyers à dix mille yuans » ou sur les « foyers spécialisés » révèlent que les uns comme les autres sont d'une taille supérieure à la moyenne. Ainsi une analyse menée en 1984 dans le district de Youbing (Sichuan) auprès de 38 familles enrichies montre-t-elle que ces dernières comptent en moyenne sept membres ${ }^{33}$. Le même type d'enquête, effectué en 1984 dans un autre district du Sichuan, révèle que les familles les plus prospères possèdent en moyenne 4,65 membres actifs ${ }^{34}$. Or, en 1982, la taille moyenne des foyers chinois était de 4,57 personnes ${ }^{35}$.

Accroître la taille de la maisonnée apparaît donc comme un moyen privilégié d'en augmenter les ressources en l'absence d'autres capitaux. Deux solutions s'offrent dès lors aux paysans : multiplier les naissances - et cette stratégie est en partie responsable de l'actuelle hausse de la natalité - , ou retarder les divisions familiales, c'est-à-dire se tourner vers des structures familiales plus complexes.

Il est certain que la famille souche, par exemple, malgré les tensions inhérentes à ce type d'organisation, permet de regrouper une force productive plus étendue, d'accumuler plus rapidement des capitaux et d'opérer par conséquent des investissements plus importants. De fait, les familles souches de notre échantillon jouissent d'un revenu annuel légèrement plus élevế que les familles nucléaires: en 1985, les premières ont réuni en moyenne un revenu global de 8875 yuans, soit 1362 yuans par personne, alors que les ressources des secondes s'élevaient à 4805 yuans, soit 1162 yuans par personne.

33. Cf. Wang Ge, « Nongcun wanyuanhu zhuanyehu jiating guanxi de diaocha » (Enquête sur les relations familiales au sein des foyers à dix mille yuans et des foyers spécialisés dans les zones rurales), Shehui, 1984, 4, pp. 26-27.

34. Cf. Wang Guoqing, « Zhuanyehu shi nongcun fazhan shangpin shengchan qinlao zhifu de daitou ren " (Les foyers spécialisés prennent la tête du mouvement rural de développement de l'économie marchande et d'enrichissement grâce à un travail ardu), Chongqing shehui kexue, 1984, pp. 56-60.

35. Cf. Mao Xiongsheng et Zhou Guangxia, « Renkou nianling jiegou dui jiating bianhua de yingxiang " (L'influence de la structure par âge de la population sur les transformations familiales), Renkou yanjiu, 1985, 5, pp. 8-12. 
Certes, on a parlé d'une recrudescence des divisions familiales lors du mouvement de redistribution des terres, et la presse chinoise dénonce aujourd'hui l'abandon fréquent des parents par leurs enfants une fois qu'ils ne peuvent plus participer au travail de production domestique ${ }^{36}$. Mais ces phénomènes'n'apparaissent pas en contradiction avec le développement de structures familiales plus complexes que les familles nucléaires, dû à une série de contraintes telles que la division des terres pour une période de quinze ans sans réelle possibilité d'adaptation à l'évolution du groupe familial et la création d'industries domestiques réclamant une main-d'œuvre abondante.

Le développement de structures familiales plus complexes ne signifie pas la multiplication des familles étendues de type traditionnel, mais plutôt le report, dans la mesure du possible, de la division familiale afin de conserver la force de travail optimum pour tirer profit des activités de production locales. Ainsi, tous les mariages conclus à Baozi Pu depuis la création de l'usine et des ateliers domestiques ont donné naissance à des familles souches au sein de l'échantillon étudié. La seule division familiale mentionnée a été opérée entre le plus jeune d'une famille de cinq fils et ses parents, âgés de 80 et 90 ans (les quatre aînés ayant quitté le toit familial à des dates antérieures). Le fils a conservé la maison familiale et l'atelier domestique, comprenant trois métiers à tisser. Il a bâti une maison pour ses parents. Ces derniers sont trop vieux pour travailler, mais ont conservé l'usufruit de la parcelle de terre attribuée à la famille avant sa division. Les cinq fils viennent travailler sur cette parcelle lors des gros travaux agricoles et donnent de l'argent à leurs parents s'ils veulent procéder à des dépenses importantes.

Dans certains cas, les familles souches qui surgissent s'éloignent des schémas traditionnels dans la mesure où, comme nous l'avons vu, le jeune couple conserve la totalité du salaire de l'époux, tout en bénéficiant des ressources tirées de l'atelier de tapis où travaille l'épouse.

36. Voir par exemple l'article de Deng Jiamin paru le 29 octobre 1987 dans la revue Zhongguo fazhi bao, p. 3. 
Que la première phase du mouvement d'industrialisation ne conduise pas nécessairement à la nucléarisation des foyers constitue un phénomène qu'ont également relevé des chercheurs travaillant sur d'autres aires géographiques. Angelique Janssens, observant l'évolution des structures familiales dans une ville des Pays-Bas entre 1880 et 1920 , écrit par exemple : «L'évolution démographique et industrielle rapide que la ville a connue pendant cette période n'a pas abouti à une remise en cause du modèle traditionnel des familles étendues... Une forte position économique et sociale semble être liée à une structure familiale complexe ${ }^{37}$. L'enquête démographique au centième réalisée en 1987 en Chine révèle d'ailleurs que les foyers regroupant les membres de trois générations sont en augmentation puisqu'ils sont passés de $17,13 \%$ en 1978 à $18,52 \%$ en $1987^{38}$. Une étude menée dans les districts ruraux de la ville de Shanghai montre enfin que les familles nucléaires se développent dans les régions exclusivement rurales, alors que les familles souches se multiplient dans les districts où la diversification des activités de production et la création d'industries domestiques sont possibles ${ }^{39}$.

Au niveau du village, l'initiative des cadres locaux et l'alliance réussie entre une industrie collective et des industries domestiques ; au niveau familial, la prépondérance accordée à l'intérêt collectif en matière de division du travail et d'utilisation des ressources : ainsi s'explique l'enrichissement rapide des paysans de Baozi Pu. L'enquête menée suggère également qu'un processus d'industrialisation n'entraîne pas aussitôt une nucléarisation des structures familiales. L'évolution de ces dernières répond, de façon plus complexe, aux critères économiques, sociaux et culturels locaux.

37. Angelique Janssens, «Industrialization without family change ? The extended family and the life cycle in a Dutch industrial town, 1880-1920 », Journal of Family History, 11, 1 (1983), pp. 25-42.

38. Cf. Renmin ribao, 5 août 1988.

39. Cf. Fu Luxia et Liu Bing, "Jiating jiegou cheng duoyanghua fazhan " (Les structures familiales se diversifient), Shehui, 1987, 5, pp. 39-41. 
Nos analyses ne pourront toutefois être confirmées que par une nouvelle étude des transformations familiales à $\mathrm{Baozi} \mathrm{Pu}$, menée par exemple dans une décennie : en effet la prospérité de cette communauté est trop récente pour que des tendances stables et durables puissent être aujourd'hui dégagées. 